\title{
MICROPULSES AND DIFFERENT TYPES OF BROWNIAN MOTION
}

\author{
MATTHIEU MAROUBY, ${ }^{*}$ Université Paul Sabatier
}

\begin{abstract}
In this paper we study sums of micropulses that generate different kinds of processes. Fractional Brownian motion and bifractional Brownian motion are obtained as limit processes. Moreover, we not only prove the convergence of finite-dimensional laws but also, in some cases, convergence in distribution in the space of right-continuous functions with left limits. Finally, we obtain generalizations with multidimensional indices.
\end{abstract}

Keywords: Sums of micropulses; fractional Brownian motion; multifractional Brownian motion; Poisson random measure

2010 Mathematics Subject Classification: Primary 60G15

Secondary 60G22; 60G60

\section{Introduction}

On the real line, a micropulse is a jump at a time $\tau$ of size $\varepsilon$ followed by a canceling jump at time $\tau+w$ of size $-\varepsilon$. In [5] and [6], Cioczek-Georges and Mandelbrot used a sum of micropulses to obtain limit processes with interesting properties, in particular fractional Brownian motion as a limit. Fractional Brownian motion (FBM) with parameter $0<H \leq 1$ is a centered Gaussian process that was introduced in [11] and developed in more detail in [12]. Its covariance function for $s, t \in \mathbb{R}_{+}$is given by

$$
r(s, t)=\frac{1}{2}\left(|t|^{2 H}+|s|^{2 H}-|t-s|^{2 H}\right) .
$$

FBM is widely used in many different areas for modeling purposes thanks to its properties (self-similarity and stationarity of increments, among others).

Micropulses were generalized as random ball models and studied by Biermé and Estrade [1], with Kaj et al. [8] studying similar models. For more recent research on random ball models, see [4] and Biermé et al. [2], who introduced a general framework for rescaled random ball models.

In this work a different approach is highlighted. We consider only micropulses, but the canceling jump may or may not be the same size as the first jump. Here, we will consider a micropulse to be given by $\left(X, X^{\prime}, \tau, w\right)$, with $\varepsilon X$ being the size of the jump at time $\tau$ and $-\varepsilon X^{\prime}$ being the size of the canceling jump at time $\tau+w$. We will sum the size of all the initial and canceling jumps occurring during a time interval.

Our aim is to study the limit process of a sum of micropulses between two times whether or not the size of the initial and canceling jumps of a micropulse is the same. Micropulses will be distributed according to a Poisson process such that, when $\varepsilon$ tends to 0 , the number of micropulses will increase and their heights will simultaneously decrease.

Received 8 October 2010; revision received 14 March 2011.

* Postal address: Institut de Mathématiques de Toulouse, Université Paul Sabatier, 118 route de Narbonne, F-31062

Toulouse Cedex 9, France. Email address: marouby@math.univ-toulouse.fr 
We will not only see that the limit process is different, but also that the scaling used to obtain a nontrivial process depends on the equality of the size of the initial jump and the canceling jump. The first model studied is almost identical to that introduced in [5], except that we allow every micropulse to have a different height for the initial and canceling jumps. The limit process will be an FBM or a standard Brownian motion. Moreover, we prove the convergence of the finite-dimensional laws, and obtain the tightness of the family of processes, which means that there is a weak convergence in the space $D$ of functions that are right continuous and have left-hand limits, which was not the case previously.

Finally, we modify and generalize the model to incorporate a multidimensional index. The limit process will be either a standard Brownian sheet or a bifractional Brownian sheet. The bifractional Brownian sheet, introduced by Tudor and Xiao [14], is the generalization of bifractional Brownian motion to a multidimensional index. Bifractional Brownian motion (BBM) motion is a centered Gaussian process introduced by Houdré and Villa [7]. This process is a generalization of FBM which keeps some of its properties (self-similarity, stationarity of small increments). BBM with parameters $(H, K)$, where $0<H \leq 1$ and $0<K \leq 1$, is a Gaussian process whose covariance function for $s, t \in \mathbb{R}_{+}$is given by

$$
r(s, t)=\frac{1}{2^{K}}\left(\left(|t|^{2 H}+|s|^{2 H}\right)^{K}-|t-s|^{2 H K}\right) .
$$

Note that, for $K=1$, this is regular FBM. The bifractional Brownian sheet is a centered Gaussian process whose covariance function for $s, t \in \mathbb{R}_{+}^{d}$ is given by

$$
r(s, t)=\prod_{i=1}^{d} \frac{1}{2^{K}}\left(\left(\left|t_{i}\right|^{2 H}+\left|s_{i}\right|^{2 H}\right)^{K}-\left|t_{i}-s_{i}\right|^{2 H K}\right)
$$

(see Section 3).

In the above two cases, the rescaling is different depending on whether the size of the initial and canceling jumps are the same or not (i.e. $\varepsilon^{1+\theta / 2}$ instead of $\varepsilon$ for example). An interpretation of this is that there is a standard Brownian motion (or sheet) and a 'noise' process that are negligible at the limit. The standard Brownian motion is due to the contribution of the micropulses which have both their initial and canceling jumps in the interval considered, whereas the underlying noise process is due to the less frequent micropulses which only have one of their jumps (initial or canceling) in the interval considered. When the size is the same, the standard Brownian motion (or sheet) disappears and, consequently, the remaining noise process becomes the main process, which explains why the scaling is different.

\section{Convergence towards Brownian motion or fractional Brownian motion}

For $\varepsilon>0$, consider the measure $n_{\varepsilon}$ on the space $E=\mathbb{R}^{3} \times \mathbb{R}_{+}$defined by

$$
n_{\varepsilon}\left(\mathrm{d} x, \mathrm{~d} x^{\prime}, \mathrm{d} \tau, \mathrm{d} w\right)=\frac{1}{2} \varepsilon^{-2} w^{-1-\theta} \mathbf{1}_{\{w>\varepsilon\}} F\left(\mathrm{~d} x, \mathrm{~d} x^{\prime}\right) \mathrm{d} \tau \mathrm{d} w,
$$

where $0<\theta<1$ and $F$ is the distribution of a random vector $\left(X, X^{\prime}\right)$ such that, for $k, l \in \mathbb{N}$, $\mathrm{E}\left[|X|^{k}\left|X^{\prime}\right|^{l}\right]<\infty$.

This measure can be seen as the intensity measure of a Poisson process $\Pi_{\varepsilon}$ on $\mathbb{R}^{3} \times \mathbb{R}_{+}$ and, consequently, of the Poisson random measure associated with $\Pi_{\varepsilon}$ that we will denote by $N_{\varepsilon}$. The size of the rise of the micropulse occurring at time $\tau$ is $\varepsilon X$, and the size of the fall occurring at time $\tau+w$ is $\varepsilon X^{\prime}$. When $\varepsilon$ goes to 0 , the height of the micropulses goes to 0 while their number goes to $\infty$. 
In this section we study the process $Y_{\varepsilon}$ defined on $\mathbb{R}_{+}$as the sum over the Poisson process $\Pi_{\varepsilon}$ (or, equivalently, the integral over the random measure $N_{\varepsilon}$ ), i.e. we will sum the rises and falls of micropulses which are taking place between 0 and $t$. We will be interested in the convergence of this process when $\varepsilon$ goes to 0 . Process $Y_{\varepsilon}$ is rigorously defined for $t \geq 0$ by

$$
Y_{\varepsilon}(t)=\sum_{j \in \mathbb{N}} \varepsilon\left(X_{j} \mathbf{1}_{\left\{0 \leq \tau_{j}<t\right\}}-X_{j}^{\prime} \mathbf{1}_{\left\{0 \leq \tau_{j}+w_{j}<t\right\}}\right)
$$

where $\left(X_{j}, X_{j}^{\prime}, \tau_{j}, w_{j}\right)$ is an enumeration of the points of the Poisson point process $\Pi_{\varepsilon}$.

Moreover, we will be able to prove weak convergence, which is a consequence of the tightness of the family of processes considered.

Theorem 2.1. (a) If $X=X^{\prime}$ almost surely then the process $Y_{\varepsilon}$ converges weakly when $\varepsilon \rightarrow 0$ to

$$
\sqrt{\frac{\mathrm{E}\left[X^{2}\right]}{\theta(1-\theta)}} B_{H},
$$

where $B_{H}$ is an FBM of Hurst parameter $H=(1-\theta) / 2$.

(b) If $X \neq X^{\prime}$ on a nonnegligible set then the process $\varepsilon^{\theta / 2}\left(Y_{\varepsilon}-\mathrm{E}\left[Y_{\varepsilon}\right]\right)$ converges weakly when $\varepsilon \rightarrow 0$ to

$$
\sqrt{\frac{\mathrm{E}\left[\left(X-X^{\prime}\right)^{2}\right]}{2 \theta}} B,
$$

where $B$ is a standard Brownian motion.

Remark 2.1. Note that the limit processes are FBMs of Hurst parameter $H<\frac{1}{2}$.

Proof of Theorem 2.1. The proof consists of two parts. First, we prove the convergence of the finite-dimensional laws, then we prove the tightness of the family $\left(Y_{\varepsilon}\right)_{\varepsilon \in[0,1)}$ or $\left(\varepsilon^{\theta / 2}\left(Y_{\varepsilon}-\right.\right.$ $\left.\left.\mathrm{E} Y_{\varepsilon}\right)\right)_{\varepsilon \in[0,1)}$.

Part 1: proof of the convergence of the finite-dimensional laws. First of all, let us introduce some notation which will be useful in the rest of the proof.

Define $A_{t}^{1}=\{(\tau, w): 0 \leq \tau<t, w>0\}$ and $A_{t}^{2}=\{(\tau, w): 0 \leq \tau+w<t, w>0\}$. We can rewrite process $Y_{\varepsilon}$ in terms of the Poisson random measure $N_{\varepsilon}$ associated with $\Pi_{\varepsilon}$ and these two sets. Indeed,

$$
Y_{\varepsilon}(t)=\varepsilon \int_{E}\left(x \mathbf{1}_{A_{t}^{1}}(\tau, w)-x^{\prime} \mathbf{1}_{A_{t}^{2}}(\tau, w)\right) N_{\varepsilon}\left(\mathrm{d} x, \mathrm{~d} x^{\prime}, \mathrm{d} \tau, \mathrm{d} w\right) .
$$

In the following we will use other sets, because it will be easier to disjoin the sets where we have complete micropulses $X-X^{\prime}$ from those where there is only $+X$ or $-X^{\prime}$.

Let us rewrite process $Y_{\varepsilon}$ using the following sets for $s, t \geq 0$ :

$$
\begin{aligned}
S_{t}^{1} & =\{(\tau, w): \tau<0,0 \leq \tau+w<t\}, \\
S_{t}^{2} & =\{(\tau, w): 0 \leq \tau<t, 0 \leq \tau+w<t\}, \\
S_{t}^{3} & =\{(\tau, w): 0 \leq \tau<t, t \leq \tau+w\}, \\
S_{s, t}^{4} & =\{(\tau, w): 0 \leq \tau<s, t \leq \tau+w\}, \\
S_{s, t}^{5} & =\{(\tau, w): 0 \leq \tau<s, s \leq \tau+w<t\} .
\end{aligned}
$$


Using these sets, we have

$$
Y_{\varepsilon}(t)=\varepsilon \int_{E}\left(x \mathbf{1}_{S_{t}^{3}}(\tau, w)-x^{\prime} \mathbf{1}_{S_{t}^{1}}(\tau, w)+\left(x-x^{\prime}\right) \mathbf{1}_{S_{t}^{2}}(\tau, w)\right) N_{\varepsilon}\left(\mathrm{d} x, \mathrm{~d} x^{\prime}, \mathrm{d} \tau, \mathrm{d} w\right) .
$$

For $0 \leq s \leq t$, we can easily obtain the following rules between the sets defined in (2.1a)(2.1e):

$$
\begin{array}{lll}
S_{s}^{1} \cap S_{t}^{1}=S_{s}^{1}, & S_{s}^{1} \cap S_{t}^{2}=\varnothing, & S_{s}^{1} \cap S_{t}^{3}=\varnothing, \\
S_{s}^{2} \cap S_{t}^{1}=\varnothing, & S_{s}^{2} \cap S_{t}^{2}=S_{s}^{2}, & S_{s}^{2} \cap S_{t}^{3}=\varnothing, \\
S_{s}^{3} \cap S_{t}^{1}=\varnothing, & S_{s}^{3} \cap S_{t}^{2}=S_{s, t}^{5}, & S_{s}^{3} \cap S_{t}^{3}=S_{s, t}^{4}, \\
S_{s, t}^{4} \cup S_{s, t}^{5}=S_{s}^{3} . & &
\end{array}
$$

In order to prove the convergence of the finite-dimensional laws, let us calculate the characteristic function $\psi_{\varepsilon}\left(\xi_{1}, \ldots, \xi_{n}\right)$ of a linear combination $\sum_{k=1}^{n} \xi_{k}\left(Y_{\varepsilon}\left(t_{k}\right)-\mathrm{E}\left[Y_{\varepsilon}\left(t_{k}\right)\right]\right)$. Note that $\mathrm{E}\left[Y_{\varepsilon}\left(t_{k}\right)\right]=0$ when $X=X^{\prime}$ almost surely.

Recall that if $N$ is a Poisson random measure on a space $E$ with intensity measure $n$ then, according to Lemma 12.2 of [9],

$$
\mathrm{E}\left[\exp \left(i \int_{E} g \mathrm{~d} N\right)\right]=\exp \left(\int_{E}\left(\mathrm{e}^{i g}-1\right) \mathrm{d} n\right) .
$$

First, let us study the case $X=X^{\prime}$. Using (2.3), we have

$$
\begin{aligned}
& \psi_{\varepsilon}\left(\xi_{1}, \ldots, \xi_{n}\right) \\
&= \mathrm{E}\left[\exp \left(i \sum_{k=1}^{n} \xi_{k} Y_{\varepsilon}\left(t_{k}\right)\right)\right] \\
&= \mathrm{E}\left[\exp i \int_{E} \varepsilon \sum_{k=1}^{n} \xi_{k} x\left(\mathbf{1}_{S_{t_{k}}^{3}}(\tau, w)-\mathbf{1}_{S_{t_{k}}^{1}}(\tau, w)\right) N_{\varepsilon}(\mathrm{d} x, \mathrm{~d} x, \mathrm{~d} \tau, \mathrm{d} w)\right] \\
&= \exp \int_{E}\left(\exp \left(i \varepsilon \sum_{k=1}^{n} \xi_{k} x\left(\mathbf{1}_{S_{t_{k}}^{3}}(\tau, w)-\mathbf{1}_{S_{t_{k}}^{1}}(\tau, w)\right)\right)-1\right) n_{\varepsilon}(\mathrm{d} x, \mathrm{~d} x, \mathrm{~d} \tau, \mathrm{d} w) \\
&= \exp \left[\int _ { E } \left(\exp \left(i \varepsilon \sum_{k=1}^{n} \xi_{k} x\left(\mathbf{1}_{S_{t_{k}}^{3}}(\tau, w)-\mathbf{1}_{S_{t_{k}}^{1}}(\tau, w)\right)\right)-1\right.\right. \\
&\left.\left.\quad-\left(i \varepsilon \sum_{k=1}^{n} \xi_{k} x\left(\mathbf{1}_{S_{t_{k}}^{3}}(\tau, w)-\mathbf{1}_{S_{t_{k}}^{1}}(\tau, w)\right)\right)\right) n_{\varepsilon}(\mathrm{d} x, \mathrm{~d} x, \mathrm{~d} \tau, \mathrm{d} w)\right]
\end{aligned}
$$

The last term on the right-hand side of (2.4) appears because $Y_{\varepsilon}$ is centered. As we have $|\exp (i x)-1-i x| \leq C x^{2}$, the integrand is bounded by

$$
C\left(i \varepsilon \sum_{k=1}^{n} \xi_{k} x\left(\mathbf{1}_{S_{t_{k}}^{3}}(\tau, w)-\mathbf{1}_{S_{t_{k}}^{1}}(\tau, w)\right)\right)^{2}
$$

Since

$$
n_{\varepsilon}(\mathrm{d} x, \mathrm{~d} x, \mathrm{~d} \tau, \mathrm{d} w)=\frac{\varepsilon^{-2}}{2} w^{-1-\theta} \mathbf{1}_{\{w>\varepsilon\}} F\left(\mathrm{~d} x, \mathrm{~d} x^{\prime}\right) \mathrm{d} \tau \mathrm{d} w
$$


the integral of the above function is in fact

$$
\int_{E} C\left(i \sum_{k=1}^{n} \xi_{k} x\left(\mathbf{1}_{S_{t_{k}}^{3}}(\tau, w)-\mathbf{1}_{S_{t_{k}}^{1}}(\tau, w)\right)\right)^{2} \frac{1}{2} w^{-1-\theta} F\left(\mathrm{~d} x, \mathrm{~d} x^{\prime}\right) \mathrm{d} \tau \mathrm{d} w .
$$

We can conclude that

$$
\begin{gathered}
\frac{1}{\varepsilon^{2}}\left(\exp \left(i \varepsilon \sum_{k=1}^{n} \xi_{k} x\left(\mathbf{1}_{S_{t_{k}}^{3}}(\tau, w)-\mathbf{1}_{S_{t_{k}}^{1}}(\tau, w)\right)\right)-1\right. \\
\left.-\left(i \varepsilon \sum_{k=1}^{n} \xi_{k} x\left(\mathbf{1}_{S_{t_{k}}^{3}}(\tau, w)-\mathbf{1}_{S_{t_{k}}^{1}}(\tau, w)\right)\right)\right)
\end{gathered}
$$

is bounded uniformly in $\varepsilon$ by

$$
C\left(\sum_{k=1}^{n} \xi_{k} x\left(\mathbf{1}_{S_{t_{k}}^{3}}(\tau, w)-\mathbf{1}_{S_{t_{k}}^{1}}(\tau, w)\right)\right)
$$

which is an integrable function with respect to $w^{-1-\theta} F\left(\mathrm{~d} x, \mathrm{~d} x^{\prime}\right) \mathrm{d} \tau \mathrm{d} w$. In order to avoid cumbersome notation, for a measure defined on $\mathbb{R} \times \mathbb{R}_{+}$, we will define $p_{\varepsilon}=w^{-1-\theta} \mathbf{1}_{\{w>\varepsilon\}} \mathrm{d} \tau \mathrm{d} w$. Hence, the Lebesgue dominated convergence theorem yields

$$
\begin{aligned}
& \lim _{\varepsilon \rightarrow 0} \psi_{\varepsilon}\left(\xi_{1}, \ldots, \xi_{n}\right) \\
& \quad=\exp \left(-\frac{\mathrm{E}\left[X^{2}\right]}{2} \int_{\mathbb{R} \times \mathbb{R}_{+}}\left(\sum_{k=1}^{n} \xi_{k}\left(\mathbf{1}_{S_{t_{k}}^{3}}(\tau, w)-\mathbf{1}_{S_{t_{k}}^{1}}(\tau, w)\right)\right)^{2} \frac{1}{2} p_{0}(\mathrm{~d} \tau, \mathrm{d} w)\right) .
\end{aligned}
$$

Using the above rules between our sets given in (2.2a)-(2.2d), the integral above can be rewritten as

$$
\begin{aligned}
\int_{\mathbb{R} \times \mathbb{R}_{+}} & \left(\sum_{k=1}^{n} \xi_{k}\left(\mathbf{1}_{S_{t_{k}}^{3}}(\tau, w)-\mathbf{1}_{S_{t_{k}}^{1}}(\tau, w)\right)\right)^{2} p_{0}(\mathrm{~d} \tau, \mathrm{d} w) \\
= & \sum_{k=1}^{n} \sum_{l=1}^{n} \xi_{k} \xi_{l}\left(p_{0}\left(S_{\min \left(t_{k}, t_{l}\right), \max \left(t_{k}, t_{l}\right)}^{4}\right)+p_{0}\left(S_{\min \left(t_{k}, t_{l}\right)}^{1}\right)\right) .
\end{aligned}
$$

We can use Lemma A.2 in Appendix A to estimate values of $p_{\varepsilon}\left(S_{t_{k}}^{i}\right)$ for $i=1,2$, or 3: the bounds used in the description of $S_{t_{k}}^{i}$ are the values $a, b, c$, and $d$ in the lemma. Then, letting $\varepsilon$ go to 0 , we obtain

$$
\begin{aligned}
\int_{\mathbb{R} \times \mathbb{R}_{+}} & \left(\sum_{k=1}^{n} \xi_{k}\left(\mathbf{1}_{S_{t_{k}}^{3}}(\tau, w)-\mathbf{1}_{S_{t_{k}}^{1}}(\tau, w)\right)\right)^{2} p_{0}(\mathrm{~d} \tau, \mathrm{d} w) \\
= & \sum_{k=1}^{n} \sum_{l=1}^{n} \xi_{k} \xi_{l} \frac{1}{\theta(1-\theta)}\left(t_{k}^{1-\theta}+t_{l}^{1-\theta}-\left|t_{k}-t_{l}\right|^{1-\theta}\right) .
\end{aligned}
$$

Then, $\lim _{\varepsilon \rightarrow 0} \psi_{\varepsilon}\left(\xi_{1}, \ldots, \xi_{n}\right)$ is the characteristic function of FBM up to a multiplicative constant. 
Now, if $X \neq X^{\prime}$ on a nonnegligible set, the method is almost the same, only there are more terms to deal with. The characteristic function of the process we are studying is now

$$
\begin{aligned}
& \psi_{\varepsilon}\left(\xi_{1}, \ldots, \xi_{n}\right) \\
&= \mathrm{E}\left[\exp \left(\left(i \sum_{k=1}^{n} \xi_{k} \varepsilon^{\theta / 2} Y_{\varepsilon}\left(t_{k}\right)\right)-\mathrm{E}\left[i \sum_{k=1}^{n} \xi_{k} \varepsilon^{\theta / 2} Y_{\varepsilon}\left(t_{k}\right)\right]\right)\right] \\
&= \mathrm{E}\left[\operatorname { e x p } i \varepsilon ^ { \theta / 2 } \int _ { E } \varepsilon \sum _ { k = 1 } ^ { n } \xi _ { k } \left(x\left(\mathbf{1}_{S_{t_{k}}^{3}}(\tau, w)+\mathbf{1}_{S_{t_{k}}^{2}}(\tau, w)\right)\right.\right. \\
&\left.\left.-x^{\prime}\left(\mathbf{1}_{S_{t_{k}}^{2}}(\tau, w)+\mathbf{1}_{S_{t_{k}}^{1}}(\tau, w)\right)\right) N_{\varepsilon}\left(\mathrm{d} x, \mathrm{~d} x^{\prime}, \mathrm{d} \tau, \mathrm{d} w\right)\right] \\
& \quad \times \exp \mathrm{E}\left[-i \sum_{k=1}^{n} \xi_{k} \varepsilon^{\theta / 2} Y_{\varepsilon}\left(t_{k}\right)\right] .
\end{aligned}
$$

Applying the same steps as earlier yields

$$
\begin{aligned}
& \psi_{\varepsilon}\left(\xi_{1}, \ldots, \xi_{n}\right) \\
& =\exp \left[\int _ { E } \left(\operatorname { e x p } \left(i \varepsilon ^ { ( \theta + 2 ) / 2 } \sum _ { k = 1 } ^ { n } \xi _ { k } \left(x\left(\mathbf{1}_{S_{t_{k}}^{3}}(\tau, w)+\mathbf{1}_{S_{t_{k}}^{2}}(\tau, w)\right)\right.\right.\right.\right. \\
& \left.\left.-x^{\prime}\left(\mathbf{1}_{S_{t_{k}}^{1}}(\tau, w)+\mathbf{1}_{S_{t_{k}}^{2}}(\tau, w)\right)\right)\right)-1 \\
& -\left(i \varepsilon ^ { ( \theta + 2 ) / 2 } \sum _ { k = 1 } ^ { n } \xi _ { k } \left(x\left(\mathbf{1}_{S_{t_{k}}^{3}}(\tau, w)+\mathbf{1}_{S_{t_{k}}^{2}}(\tau, w)\right)\right.\right. \\
& \left.\quad \times n_{\varepsilon}\left(\mathrm{d} x, \mathrm{~d} x^{\prime}, \mathrm{d} \tau, \mathrm{d} w\right)\right] .
\end{aligned}
$$

Unfortunately, the Lebesgue dominated convergence theorem cannot be used because the integral is not finite when $w$ is close to 0 . Define

$$
f\left(x, x^{\prime}, \tau, w\right)=\sum_{k=1}^{n} \xi_{k}\left(x\left(\mathbf{1}_{S_{t_{k}}^{3}}(\tau, w)+\mathbf{1}_{S_{t_{k}}^{2}}(\tau, w)\right)-x^{\prime}\left(\mathbf{1}_{S_{t_{k}}^{1}}(\tau, w)+\mathbf{1}_{S_{t_{k}}^{2}}(\tau, w)\right)\right)
$$

and

$$
g_{\varepsilon}\left(x, x^{\prime}, \tau, w\right)=\frac{1}{\varepsilon^{2+\theta}}\left(\mathrm{e}^{i \varepsilon^{(\theta+2) / 2} f}-1-i \varepsilon^{(\theta+2) / 2} f\right),
$$

so that

$$
\psi_{\varepsilon}\left(\xi_{1}, \ldots, \xi_{n}\right)=\exp \int_{E} \varepsilon^{\theta} g_{\varepsilon} \mathbf{1}_{\{w>\varepsilon\}} w^{-1-\theta} F\left(\mathrm{~d} x, \mathrm{~d} x^{\prime}\right) \mathrm{d} \tau \mathrm{d} w .
$$

Furthermore, define

$$
h\left(x, x^{\prime}, \tau, w\right)=\left(x-x^{\prime}\right)^{2} \sum_{k=1}^{n} \sum_{l=1}^{n} \xi_{k} \xi_{l} \mathbf{1}_{S_{\min \left(t_{k}, t_{l}\right)}^{2}}(\tau, w)
$$


and consider

$$
\int_{E}\left(\varepsilon^{\theta} g_{\varepsilon}\left(x, x^{\prime}, \tau, w\right)+\frac{1}{2} \varepsilon^{\theta} h\left(x, x^{\prime}, \tau, w\right)\right) \mathbf{1}_{\{w>\varepsilon\}} w^{-1-\theta} F\left(\mathrm{~d} x, \mathrm{~d} x^{\prime}\right) \mathrm{d} \tau \mathrm{d} w .
$$

Note that

$$
\left|\varepsilon^{\theta} g_{\varepsilon}\left(x, x^{\prime}, \tau, w\right)+\frac{1}{2} \varepsilon^{\theta} h\left(x, x^{\prime}, \tau, w\right)\right| \leq \varepsilon^{\theta}\left|g_{\varepsilon}+\frac{1}{2} f^{2}\right|+\frac{\varepsilon^{\theta}}{2}\left|f^{2}-h\right| .
$$

On the one hand, we have

$$
g_{\varepsilon}+\frac{1}{2} f^{2}=\frac{1}{\varepsilon^{\theta+2}}\left(\mathrm{e}^{i \varepsilon^{(\theta+2) / 2} f}-1-i \varepsilon^{(\theta+2) / 2} f+\frac{\varepsilon^{\theta+2}}{2} f^{2}\right) .
$$

This implies that

$$
\varepsilon^{\theta}\left|g_{\varepsilon}+\frac{1}{2} f^{2}\right| \leq C \varepsilon^{3 \theta / 2+1}|f|^{3} .
$$

However,

$$
\begin{aligned}
f^{3}=\sum_{j, k, l=1}^{n} \xi_{j} \xi_{k} \xi_{l} & \left(x^{3} \mathbf{1}_{S_{\min \left(t_{j}, t_{k}, t_{l}\right), \max \left(t_{j}, t_{k}, t_{l}\right)}^{4}}+\left(x-x^{\prime}\right)^{3} \mathbf{1}_{S_{\min \left(t_{j}, t_{k}, t_{l}\right)}^{2}}\right. \\
& +x^{2}\left(x-x^{\prime}\right)\left(\mathbf{1}_{\left\{S_{t_{j}}^{1} \cap S_{t_{k}}^{1} \cap S_{t_{l}}^{2}\right\}}+\mathbf{1}_{\left\{S_{t_{j}}^{1} \cap S_{t_{k}}^{2} \cap S_{t_{l}}^{1}\right\}}+\mathbf{1}_{\left\{S_{t_{j}}^{2} \cap S_{t_{k}}^{1} \cap S_{t_{l}}^{1}\right\}}\right) \\
& +x\left(x-x^{\prime}\right)^{2}\left(\mathbf{1}_{\left\{S_{t_{j}}^{1} \cap S_{t_{k}}^{2} \cap S_{t_{l}}^{2}\right\}}+\mathbf{1}_{\left\{S_{t_{j}}^{2} \cap S_{t_{k}}^{1} \cap S_{t_{l}}^{2}\right\}}+\mathbf{1}_{\left\{S_{t_{j}}^{2} \cap S_{t_{k}}^{2} \cap S_{t_{l}}^{1}\right\}}\right) \\
& -x^{\prime 3} \mathbf{1}_{\left.S_{\min \left(t_{j}, t_{k}, t_{l}\right)}^{1}\right) .}
\end{aligned}
$$

On the other hand, using the set rules given in (2.2a)-(2.2d), expanding $f^{2}-h$ yields

$$
f^{2}-h=\sum_{k=1}^{n} \sum_{l=1}^{n} \xi_{k} \xi_{l}\left(x^{2} \mathbf{1}_{S_{\min \left(t_{k}, t_{l}\right), \max \left(t_{k}, t_{l}\right)}^{4}}+x^{\prime 2} \mathbf{1}_{S_{\min \left(t_{k}, t_{l}\right)}^{1}}\right) .
$$

Now note that, according to Lemma A.2 in Appendix A and (2.6), we have

$$
\int_{E}|f|^{3} w^{-1-\theta} \mathbf{1}_{\{w>\varepsilon\}} F\left(\mathrm{~d} x, \mathrm{~d} x^{\prime}\right) \mathrm{d} \tau \mathrm{d} w=C_{t_{1}, \ldots, t_{n}} \varepsilon^{-\theta},
$$

where $C_{t_{1}, \ldots, t_{n}}$ is a constant depending on $t_{1}, \ldots, t_{n}$ and not on $\varepsilon$. In (2.7), Lemma A.2 yields

$$
\int_{E}\left|f^{2}-h\right| w^{-1-\theta} \mathbf{1}_{\{w>\varepsilon\}} F\left(\mathrm{~d} x, \mathrm{~d} x^{\prime}\right) \mathrm{d} \tau \mathrm{d} w=C_{t_{1}, \ldots, t_{n}}^{\prime},
$$

where $C_{t_{1}, \ldots, t_{n}}^{\prime}$ does not depend on $\varepsilon$.

Using these two upper bounds in (2.5), we obtain

$$
\lim _{\varepsilon \rightarrow 0} \int_{E}\left(\varepsilon^{\theta} g_{\varepsilon}\left(x, x^{\prime}, \tau, w\right)+\frac{1}{2} \varepsilon^{\theta} h\left(x, x^{\prime}, \tau, w\right)\right) \mathbf{1}_{\{w>\varepsilon\}} w^{-1-\theta} F\left(\mathrm{~d} x, \mathrm{~d} x^{\prime}\right) \mathrm{d} \tau \mathrm{d} w=0 .
$$

In other words,

$$
\begin{aligned}
& \lim _{\varepsilon \rightarrow 0} \int_{E} \varepsilon^{\theta} g_{\varepsilon}\left(x, x^{\prime}, \tau, w\right) \mathbf{1}_{\{w>\varepsilon\}} w^{-1-\theta} F\left(\mathrm{~d} x, \mathrm{~d} x^{\prime}\right) \mathrm{d} \tau \mathrm{d} w \\
& \quad=\lim _{\varepsilon \rightarrow 0}-\frac{\varepsilon^{\theta}}{2} \int_{E} h\left(x, x^{\prime}, \tau, w\right) \mathbf{1}_{\{w>\varepsilon\}} w^{-1-\theta} F\left(\mathrm{~d} x, \mathrm{~d} x^{\prime}\right) \mathrm{d} \tau \mathrm{d} w .
\end{aligned}
$$


Using Lemma A.2, we can compute the above integral:

$$
\begin{aligned}
& \lim _{\varepsilon \rightarrow 0} \int_{E} \varepsilon^{\theta} g_{\varepsilon} \mathbf{1}_{\{w>\varepsilon\}} w^{-1-\theta} F\left(\mathrm{~d} x, \mathrm{~d} x^{\prime}\right) \mathrm{d} \tau \mathrm{d} w \\
& =-\frac{\mathrm{E}\left[\left(X-X^{\prime}\right)^{2}\right]}{4 \theta} \sum_{k=1}^{n} \sum_{l=1}^{n} \xi_{k} \xi_{l} \min \left(t_{k}, t_{l}\right) .
\end{aligned}
$$

Thus,

$$
\lim _{\varepsilon \rightarrow 0} \psi_{\varepsilon}\left(\xi_{1}, \ldots, \xi_{n}\right)=\exp -\left(\frac{\mathrm{E}\left[\left(X-X^{\prime}\right)^{2}\right]}{4 \theta} \sum_{k=1}^{n} \sum_{l=1}^{n} \xi_{k} \xi_{l} \min \left(t_{k}, t_{l}\right)\right),
$$

which is the characteristic function of a standard Brownian motion up to a multiplicative constant.

Part 2: proof of the tightness. In this part of the proof, $C$ will denote a generic constant that may change from line to line. The studied processes are in the space $D$ of real-valued functions on $[0,1]$ that are right continuous and have left-hand limits. According to Theorem 13.5 of [3], tightness of the family $\left(Y_{\varepsilon}(t)\right)_{0<\varepsilon<1}$ is proven if there exist $n \in \mathbb{N}$ and $\gamma>1$ such that, for $s \leq t \leq u$,

$$
\mathrm{E}\left[\left|Y_{\varepsilon}(t)-X_{\varepsilon}(s)\right|^{n}\left|Y_{\varepsilon}(u)-Y_{\varepsilon}(t)\right|^{n}\right] \leq C|u-s|^{\gamma} .
$$

The objective is to obtain a bound from above as in (2.8), which will be achieved using Lemma A.1 in Appendix A. For each term appearing in Lemma A.1, we will lower bound the highest power $\beta$ possible in the bound of each $I_{l, k}$ by $C(u-s)^{\beta}$.

In this part, we will use the sets introduced in the finite-dimensional proof. Recall that $A_{t}^{1}=\{(\tau, w): 0 \leq \tau<t, w>0\}$ and $A_{t}^{2}=\{(\tau, w): 0 \leq \tau+w<t, w>0\}$. Define, for $s<t, B_{s, t}^{1}=A_{t}^{1} \backslash A_{s}^{1}$ and $B_{s, t}^{2}=A_{t}^{2} \backslash A_{s}^{2}$. Then $B_{s, t}^{1}=\{(\tau, w): s \leq \tau<t, w>0\}$ and $B_{s, t}^{2}=\{(\tau, w): s \leq \tau+w<t, w>0\}$. As $x \mathbf{1}_{A_{t}^{1}}(\tau, w)-x^{\prime} \mathbf{1}_{A_{t}^{2}}(\tau, w) \in L^{1}\left(n_{\varepsilon}\right)$, we can write the increment of $Y_{\varepsilon}$ between $s$ and $t$ as

$$
Y_{\varepsilon}(t)-Y_{\varepsilon}(s)=\varepsilon \int_{E}\left(x \mathbf{1}_{B_{s, t}^{1}}(\tau, w)-x^{\prime} \mathbf{1}_{B_{s, t}^{2}}(\tau, w)\right) N_{\varepsilon}(\mathrm{d} x, \mathrm{~d} \tau, \mathrm{d} w) .
$$

Let $0 \leq s<t<u$, and define

$$
f_{1}\left(x, x^{\prime}, \tau, w\right)=\varepsilon\left(x \mathbf{1}_{B_{s, t}^{1}}(\tau, w)-x^{\prime} \mathbf{1}_{B_{s, t}^{2}}(\tau, w)\right)
$$

and

$$
f_{2}\left(x, x^{\prime}, \tau, w\right)=\varepsilon\left(x \mathbf{1}_{B_{t, u}^{1}}(\tau, w)-x^{\prime} \mathbf{1}_{B_{t, u}^{2}}(\tau, w)\right) .
$$

We are ready to apply Lemma A. 1 with $f_{1}$ and $f_{2}$ defined above and $\Pi_{\varepsilon}$ the Poisson process $\left(X_{j}, X_{j}^{\prime}, \tau_{j}, w_{j}\right)_{j \in \mathbb{N}}$. The assumptions are those of Lemma A.1 (note that condition (A.1) is satisfied because we set $\left.\mathrm{E}\left[|X|^{k}\left|X^{\prime}\right|^{l}\right]<\infty\right)$. Thus, we have to evaluate the following integrals, denoted by $I_{k, l}$ :

$$
\begin{aligned}
I_{l, k}=\varepsilon^{l+k} \int_{E} & \left(x \mathbf{1}_{B_{s, t}^{1}}(\tau, w)-x^{\prime} \mathbf{1}_{B_{s, t}^{2}}(\tau, w)\right)^{l}\left(x \mathbf{1}_{B_{t, u}^{1}}(\tau, w)-x^{\prime} \mathbf{1}_{B_{t, u}^{2}}(\tau, w)\right)^{k} \\
& \times n_{\varepsilon}\left(\mathrm{d} x, \mathrm{~d} x^{\prime}, \mathrm{d} \tau, \mathrm{d} w\right) .
\end{aligned}
$$


Note that $B_{s, t}^{1} \cap B_{t, u}^{1}=B_{s, t}^{2} \cap B_{t, u}^{2}=B_{t, u}^{1} \cap B_{s, t}^{2}=\varnothing$. Consequently, for $k \neq 0$ and $l \neq 0$, when we develop the product in the integrand, the only nonvanishing term is $x^{l}\left(-x^{\prime}\right)^{k} \mathbf{1}_{B_{s, t}^{1}} \mathbf{1}_{B_{t, u}^{2}}$. Then we obtain, for $k>0$ and $l>0$,

$$
I_{l, k}=\frac{1}{2} \varepsilon^{k+l-2} \mathrm{E}\left[X^{l}\left(-X^{\prime}\right)^{k}\right] p_{\varepsilon}\left(B_{s, t}^{1} \cap B_{t, u}^{2}\right) .
$$

Let us bound from above $\varepsilon^{k+l-2} p_{\varepsilon}\left(B_{s, t}^{1} \cap B_{t, u}^{2}\right)$. As $B_{s, t}^{1} \cap B_{t, u}^{2}=\{(\tau, w): s \leq \tau<t, t \leq$ $\tau+w<u\}$, using Lemma A.3 in Appendix A,

- if $u-s \leq \varepsilon$ then $\varepsilon^{k+l-2} p_{\varepsilon}\left(B_{s, t}^{1} \cap B_{t, u}^{2}\right)=0$,

- if $u-s>\varepsilon$ then $\varepsilon^{k+l-2} p_{\varepsilon}\left(B_{s, t}^{1} \cap B_{t, u}^{2}\right) \leq(2 / \theta)(u-s)^{k+l-1-\theta}$.

To sum up,

$$
I_{l, k} \leq \frac{2}{\theta(1-\theta)}(u-s)^{k+l-1-\theta} .
$$

We still have to compute $I_{l, 0}$ and $I_{0, k}$. As the computations are the same, we only provide details for the $I_{l, 0}$ integral.

From now on, we will have to distinguish between the cases when $X \neq X^{\prime}$ on a nonnegligible set and $X=X^{\prime}$ almost surely.

Case (a): $X=X^{\prime}$ almost surely. We have

$$
I_{l, 0}=\varepsilon^{l} \int_{E} x^{l}\left(\mathbf{1}_{B_{s, t}^{1}}(\tau, w)-\mathbf{1}_{B_{s, t}^{2}}(\tau, w)\right)^{l} \mathrm{~d} n_{\varepsilon}\left(x, x^{\prime}, \tau, w\right) .
$$

By expanding this expression,

$$
I_{l, 0}=\frac{\varepsilon^{l-2}}{2} \mathrm{E}\left[X^{l}\right] \sum_{i=0}^{l}\left(\begin{array}{l}
l \\
i
\end{array}\right)(-1)^{l-i} \int \mathbf{1}_{B_{s, t}^{1}}(\tau, w)^{i} \mathbf{1}_{B_{s, t}^{2}}(\tau, w)^{l-i} \mathrm{~d} p_{\varepsilon}(\tau, w) .
$$

Note that, for $0<i<l$, the integral above does not depend on $i$. For $i=0$,

$$
\int \mathbf{1}_{B_{s, t}^{2}}(\tau, w)^{l} \mathrm{~d} p_{\varepsilon}(\tau, w)=p_{\varepsilon}\left(B_{s, t}^{2}\right)=p_{\varepsilon}\left(B_{s, t}^{2} \cap B_{s, t}^{1}\right)+p_{\varepsilon}\left(B_{s, t}^{2} \cap\left(B_{s, t}^{1}\right)^{\mathrm{c}}\right),
$$

where $A^{\mathrm{c}}$ is the complement of the set $A$ in $\mathbb{R} \times \mathbb{R}_{+}$. For $i=l$, in the same way, we have

$$
\int \mathbf{1}_{B_{s, t}^{1}}(\tau, w)^{l} \mathrm{~d} p_{\varepsilon}(\tau, w)=p_{\varepsilon}\left(B_{s, t}^{1}\right)=p_{\varepsilon}\left(B_{s, t}^{1} \cap B_{s, t}^{2}\right)+p_{\varepsilon}\left(B_{s, t}^{1} \cap\left(B_{s, t}^{2}\right)^{\mathrm{c}}\right) .
$$

Then, (2.10) becomes

$$
\begin{gathered}
I_{l, 0}=\frac{\varepsilon^{l-2}}{2} \mathrm{E}\left[X^{l}\right]\left(p_{\varepsilon}\left(B_{s, t}^{1} \cap B_{s, t}^{2}\right) \sum_{i=0}^{l}\left(\begin{array}{l}
l \\
i
\end{array}\right)(-1)^{l-i}+p_{\varepsilon}\left(B_{s, t}^{2} \cap\left(B_{s, t}^{1}\right)^{\mathrm{c}}\right)\right. \\
\left.+(-1)^{l} p_{\varepsilon}\left(B_{s, t}^{1} \cap\left(B_{s, t}^{2}\right)^{\mathrm{c}}\right)\right) .
\end{gathered}
$$

According to Lemma A.2 we have $p_{\varepsilon}\left(B_{s, t}^{1} \cap\left(B_{s, t}^{2}\right)^{\mathrm{c}}\right)=p_{\varepsilon}\left(B_{s, t}^{2} \cap\left(B_{s, t}^{1}\right)^{\mathrm{c}}\right)$, and as

$$
\sum_{i=0}^{l}\left(\begin{array}{l}
l \\
i
\end{array}\right)(-1)^{l-i}=0
$$


we can deduce that

$$
I_{l, 0}= \begin{cases}\varepsilon^{l-2} \mathrm{E}\left[X^{l}\right] p_{\varepsilon}\left(B_{s, t}^{1} \backslash B_{s, t}^{2}\right) & \text { if } l \text { is even, } \\ 0 & \text { if } l \text { is odd. }\end{cases}
$$

Note that $B_{s, t}^{1} \cap\left(B_{s, t}^{2}\right)^{\mathrm{c}}=\{(\tau, w): s \leq \tau<t, t \leq \tau+w\}$. When $l=2$ and $t-s>\varepsilon$, Lemma A.2 yields

$$
p_{\varepsilon}\left(B_{s, t}^{1} \cap\left(B_{s, t}^{2}\right)^{\mathrm{c}}\right)=\frac{\varepsilon^{1-\theta}}{\theta}+\frac{1}{\theta(1-\theta)}\left((t-s)^{1-\theta}-\varepsilon^{1-\theta}\right)<\frac{1}{\theta(1-\theta)}(t-s)^{1-\theta} .
$$

Otherwise, Lemma A.3 is enough to obtain interesting bounds. If $l \geq 3$ then

$$
\varepsilon^{l-2} p_{\varepsilon}\left(B_{s, t}^{1} \cap\left(B_{s, t}^{2}\right)^{\mathrm{c}}\right) \leq \frac{\varepsilon^{l-2-\theta}}{\theta}(t-s) \leq \frac{t-s}{\theta} .
$$

If $l=2$ and $t-s<\varepsilon$, then the same lemma yields

$$
p_{\varepsilon}\left(B_{s, t}^{1} \cap\left(B_{s, t}^{2}\right)^{\mathrm{c}}\right) \leq \frac{\varepsilon^{-\theta}}{\theta}(t-s) \leq \frac{(t-s)^{1-\theta}}{\theta} .
$$

To sum up, we have

$$
I_{l, 0} \leq \begin{cases}C \max \left((u-s),(u-s)^{l-\theta-1}\right) & \text { if } l \text { is even and } l>2 \\ C(u-s)^{l-1-\theta} & \text { if } l=2 \\ 0 & \text { if } l \text { is odd }\end{cases}
$$

Case $(b): X \neq X^{\prime}$ on a nonnegligible set. Remember that this is the second case of Theorem 2.1 so there is a coefficient of $\varepsilon^{\theta / 2}$ multiplying the process. Therefore, in this case we will be interested in $\tilde{I}_{l, k}$, which we use to denote $\varepsilon^{\theta(k+l) / 2} I_{l, k}$. When $k \neq 0$ and $l \neq 0$, the previous calculation we carried out to obtain (2.9) is still valid because $\varepsilon \leq 1$, i.e.

$$
\tilde{I}_{l, k} \leq \frac{2 \varepsilon^{(k+l) \theta / 2}}{\theta(1-\theta)}(u-s)^{k+l-1-\theta} \leq \frac{2}{\theta(1-\theta)}(u-s)^{k+l-1-\theta} .
$$

When $k=0$,

$$
\tilde{I}_{l, 0}=\varepsilon^{l(1+\theta / 2)-2} \int_{E}\left(x \mathbf{1}_{B_{s, t}^{1}}(\tau, w)-x^{\prime} \mathbf{1}_{B_{s, t}^{2}}(\tau, w)\right)^{l} \mathrm{~d} n\left(x, x^{\prime}, \tau, w\right) .
$$

Expanding this expression, we obtain

$$
\tilde{I}_{l, 0}=\varepsilon^{l(1+\theta / 2)-2}\left(\sum_{i=1}^{l-1}\left(\begin{array}{l}
l \\
i
\end{array}\right) \frac{\mathrm{E}\left[X^{i}\left(-X^{\prime}\right)^{l-i}\right]}{2} p_{\varepsilon}\left(B_{s, t}^{1} \cap B_{s, t}^{2}\right)+p_{\varepsilon}\left(B_{s, t}^{1}\right)+p_{\varepsilon}\left(B_{s, t}^{2}\right)\right) .
$$

Thanks to Lemma A.3, we have $p_{\varepsilon}\left(B_{s, t}^{1} \cap B_{s, t}^{2}\right)=0$ if $t-s<\varepsilon$, and if $t-s>\varepsilon$,

$$
\varepsilon^{l(1+\theta / 2)-2} p_{\varepsilon}\left(B_{s, t}^{1} \cap B_{s, t}^{2}\right) \leq \frac{\varepsilon^{l(1+\theta / 2)-2}}{\theta}(t-s) \varepsilon^{-\theta} .
$$


Remember that we are interested in elements of $s_{n}$, where $s_{n}$ is as defined in Lemma A.1 in Appendix A. If $(l, 0)$ appears in $\wp_{n}$ then $l \geq 2$. This implies that

$$
\varepsilon^{l(1+\theta / 2)-2} p_{\varepsilon}\left(B_{s, t}^{1} \cap B_{s, t}^{2}\right) \leq \frac{2 \varepsilon^{l-2}}{\theta}(t-s) \leq \frac{2(t-s)^{l-1}}{\theta} .
$$

Using Lemma A.2, we have $p_{\varepsilon}\left(B_{s, t}^{1}\right)=p_{\varepsilon}\left(B_{s, t}^{2}\right)$, and using Lemma A.3, we obtain

$$
\varepsilon^{l(1+\theta / 2)-2} p_{\varepsilon}\left(B_{s, t}^{1}\right)=\frac{\varepsilon^{l(1+\theta / 2)-2-\theta}}{\theta}(t-s) \leq \frac{1}{\theta}(t-s) .
$$

To conclude this second case, we can see that, for $l>0$ and $k>0, \tilde{I}_{l, k} \leq(u-s)^{(k+l)-1-\theta}$, otherwise $\tilde{I}_{l, 0} \leq C(u-s)$ and $\tilde{I}_{0, k} \leq C(u-s)$.

For every pair $(l, k)$ of nonnegative integers, we thus have either $I_{l, k} \leq C(u-s)^{k+l-1-\theta}$ or $I_{l, k} \leq C(u-s)$, with the same statement holding for $\tilde{I}_{l, k}$ because $\varepsilon \leq 1$.

Let us evaluate the product

$$
\prod_{i=1}^{d} \frac{\left(\begin{array}{c}
k_{i}+l_{i} \\
l_{i}
\end{array}\right)}{d !\left(k_{i}+l_{i}\right) !} I_{l_{i}, k_{i}}
$$

with $\left(l_{1}, \ldots, l_{d}, k_{1}, \ldots, k_{d}\right) \in \wp_{n}$. Recall that

$$
s_{n}=\bigcup_{d=1}^{n}\left\{k, l \in \mathbb{N}^{d}, \sum_{i=1}^{d} k_{i}=\sum_{i=1}^{d} l_{i}=n \text { and } k_{i}+l_{i} \geq 2\right\} .
$$

Using the upper bounds we obtained earlier for $I_{l, k}$, we have

$$
\prod_{i=1}^{d} \frac{\left(\begin{array}{c}
k_{i}+l_{i} \\
l_{i}
\end{array}\right)}{d !\left(k_{i}+l_{i}\right) !} I_{l_{i}, k_{i}} \leq C(u-s)^{\beta},
$$

with $\beta=\sum_{i=1}^{d} \beta_{l_{i}, k_{i}}$, where the $\beta_{l_{i}, k_{i}}$ are the upper bounds of the power we just obtained for $I_{l_{i}, k_{i}} \leq C(u-s)^{\beta_{l_{i}, k_{i}}}$. Let us show that $\beta>1$.

- If, for some $i, \beta_{l_{i}, k_{i}}=1$ then $k_{i}=0$ or $l_{i}=0$. Consequently, as $\sum_{i=1}^{d} k_{i}=\sum_{i=1}^{d} l_{i}=n$, then we must have $d \geq 2$. This implies that $\beta>\beta_{l_{i}, k_{i}}=1$.

- If, for all $i, \beta_{l_{i}, k_{i}}=k_{i}+l_{i}-1-\theta$ then

$$
\beta=\sum k_{i}+l_{i}-1-\theta=2 n-d(1+\theta) .
$$

As $d \leq n$, then

$$
\beta \geq n(1-\theta)
$$

If we take $n>1 /(1-\theta)$ then $\beta>1$.

Consequently, for $n>1 /(1-\theta)$, there exists $\beta>1$ such that

$$
\prod_{i=1}^{d} \frac{\left(\begin{array}{c}
k_{i}+l_{i} \\
l_{i}
\end{array}\right)}{d !\left(k_{i}+l_{i}\right) !} I_{l_{i}, k_{i}} \leq C(u-s)^{\beta} .
$$

As the above holds for $\tilde{I}_{l, k}$ in place of $I_{l, k}$, the tightness criterion is proved.

This completes the proof of Theorem 2.1 . 


\section{Convergence towards a Brownian sheet or a bifractional Brownian sheet}

This section will be devoted to the study of processes indexed by $t=\left(t^{1}, \ldots, t^{d}\right) \in \mathbb{R}_{+}^{d}$ for $d \geq 2$.

In this section we will obtain a bifractional Brownian sheet as a limit process. The bifractional Brownian sheet is a Gaussian process with parameters $(H, K)$ whose covariance function for $t=\left(t^{1}, \ldots, t^{d}\right), s=\left(s^{1}, \ldots, s^{d}\right) \in \mathbb{R}_{+}^{d}$ is given by

$$
\frac{1}{2^{d K}} \prod_{i=1}^{d}\left|\left(t^{i}\right)^{2 H}+\left(s^{i}\right)^{2 H}\right|^{K}-\left|t^{i}-s^{i}\right|^{2 H K} .
$$

Let us consider the Poisson random measure $M_{\varepsilon}$ defined through its intensity measure on $E=\mathbb{R} \times \mathbb{R} \times \mathbb{R}^{d} \times \mathbb{R}_{+}^{d}$ by

$$
\mu_{\varepsilon}\left(\mathrm{d} x, \mathrm{~d} x^{\prime}, \mathrm{d} \tau, \mathrm{d} w\right)=\frac{1}{2} \varepsilon^{-2} \prod_{i=1}^{d}\left(w_{i}^{-1-\theta} \mathbf{1}_{\left\{w_{i}>\varepsilon\right\}} \mathrm{d} \tau_{i} \mathrm{~d} w_{i}\right) F\left(\mathrm{~d} x, \mathrm{~d} x^{\prime}\right)
$$

with $0<\theta<1$ and $F$ as in the previous section. Moreover, denote by $\Pi_{\varepsilon}^{\prime}$ the Poisson point process associated with $M_{\varepsilon}$. For $j \in\{1,2,3\}$, consider sets $\mathbb{S}_{t}^{j}=\prod_{i=1}^{d} \tilde{S}_{t^{i}}^{j}$, where the sets $\tilde{S}_{t^{i}}^{j}$ are subsets of $\mathbb{R}^{2}$ defined for $t>0$ by

$$
\begin{aligned}
& \tilde{S}_{t}^{1}=\{(\tau, w): \tau<-t,-t \leq \tau+w<t\}, \\
& \tilde{S}_{t}^{2}=\{(\tau, w):-t \leq \tau<t,-t \leq \tau+w<t\}, \\
& \tilde{S}_{t}^{3}=\{(\tau, w):-t \leq \tau<t, t \leq \tau+w\} .
\end{aligned}
$$

Consider the process defined for $t \in \mathbb{R}_{+}^{d}$ by

$$
\breve{Y}_{\varepsilon}(t)=\sum_{j \in \mathbb{N}} \varepsilon\left(X_{j} \mathbf{1}_{\left(\tau_{j}, w_{j}\right) \in \mathbb{S}_{t}^{3}}-X_{j}^{\prime} \mathbf{1}_{\left(\tau_{j}, w_{j}\right) \in \mathbb{S}_{t}^{1}}+\left(X-X^{\prime}\right) \mathbf{1}_{\mathbb{S}_{t}^{2}}\left(\tau_{j}, w\right)\right),
$$

where $\left(X_{j}, X_{j}^{\prime}, \tau_{j}, w_{j}\right)_{j \in \mathbb{N}}$ is an enumeration of the Poisson point process $\Pi_{\varepsilon}^{\prime}$.

Theorem 3.1. (a) If $X=X^{\prime}$ almost surely then the finite-dimensional laws of $\breve{Y}_{\varepsilon}$ converge to those of

$$
\sqrt{\frac{2^{d(1-\theta)-1} \mathrm{E}\left[X^{2}\right]}{\theta^{d}(1-\theta)^{d}}} B_{H, K},
$$

where $B_{(H, K)}$ is a bifractional Brownian sheet with parameters $(H, K)=\left(\frac{1}{2}, 1-\theta\right)$.

(b) If $X \neq X^{\prime}$ on a nonnegligible set, the finite-dimensional laws of the process $\varepsilon^{d \theta / 2}\left(\breve{Y}_{\varepsilon}(t)-\right.$ $\left.\mathrm{E}\left[\breve{Y}_{\varepsilon}\right]\right)$ converge to those of

$$
\sqrt{\frac{2^{d-1} \mathrm{E}\left[\left(X-X^{\prime}\right)^{2}\right]}{\theta^{d}}} B
$$

where $B$ is a Brownian sheet.

Proof. (a) The process $\breve{Y}_{\varepsilon}$ can be written as

$$
\breve{Y}_{\varepsilon}(t)=\varepsilon \int_{E} x\left(\mathbf{1}_{\mathbb{S}_{t}^{3}}(\tau, w)-\mathbf{1}_{\mathbb{S}_{t}^{1}}(\tau, w)\right) M_{\varepsilon}\left(\mathrm{d} x, \mathrm{~d} x^{\prime}, \mathrm{d} \tau, \mathrm{d} w\right) .
$$


We can write

$$
\breve{Z}_{\varepsilon}^{1}(t)=\varepsilon \int_{E} x \mathbf{1}_{\mathbb{S}_{t}^{1}}(\tau, w) N_{\varepsilon}\left(\mathrm{d} x, \mathrm{~d} x^{\prime}, \mathrm{d} \tau, \mathrm{d} w\right)-\varepsilon \int_{E} x \mathbf{1}_{\mathbb{S}_{t}^{1}}(\tau, w) \mu_{\varepsilon}\left(\mathrm{d} x, \mathrm{~d} x^{\prime}, \mathrm{d} \tau, \mathrm{d} w\right)
$$

and

$$
\breve{Z}_{\varepsilon}^{3}(t)=\varepsilon \int_{E} x \mathbf{1}_{\mathbb{S}_{t}^{3}}(\tau, w) N_{\varepsilon}\left(\mathrm{d} x, \mathrm{~d} x^{\prime}, \mathrm{d} \tau, \mathrm{d} w\right)-\varepsilon \int_{E} x \mathbf{1}_{\mathbb{S}_{t}^{3}}(\tau, w) \mu_{\varepsilon}\left(\mathrm{d} x, \mathrm{~d} x^{\prime}, \mathrm{d} \tau, \mathrm{d} w\right),
$$

both of which are centered processes. They are independent because, for every $s, t \in \mathbb{R}_{+}^{d}$, $\mathbb{S}_{s}^{1} \cap \mathbb{S}_{t}^{3}=\varnothing$. Finally, Lemma A.2 in Appendix A can show that

$$
\int_{E} x \mathbf{1}_{\mathbb{S}_{t}^{1}}(\tau, w) \mu_{\varepsilon}\left(\mathrm{d} x, \mathrm{~d} x^{\prime}, \mathrm{d} \tau, \mathrm{d} w\right)=\int_{E} x \mathbf{1}_{\mathbb{S}_{t}^{3}}(\tau, w) \mu_{\varepsilon}\left(\mathrm{d} x, \mathrm{~d} x^{\prime}, \mathrm{d} \tau, \mathrm{d} w\right) .
$$

Then,

$$
\breve{Y}_{\varepsilon}=\breve{Z}_{\varepsilon}^{3}-\breve{Z}_{\varepsilon}^{1} .
$$

Now we will show that the finite-dimensional laws of $\breve{Z}_{\varepsilon}^{3}$ converge to those of a bifractional Brownian sheet. The same statement holds for $\breve{Z}_{\varepsilon}^{1}$, but as the proof is the same as for $\breve{Z}_{\varepsilon}^{3}$, we omit the details.

Denote by $\psi_{\varepsilon}\left(\xi_{1}, \ldots, \xi_{n}\right)$ the characteristic function of $\breve{Z}_{\varepsilon}^{3}$. Using (2.3), we have

$$
\begin{aligned}
\psi_{\varepsilon}\left(\xi_{1}, \ldots, \xi_{n}\right)=\exp \left(\int_{E}(\right. & \exp \left(i \varepsilon \sum_{k=1}^{n} \xi_{k} x \mathbf{1}_{\mathbb{S}_{t_{k}}^{3}}(\tau, w)\right)-1 \\
& \left.\left.\quad\left(i \varepsilon \sum_{k=1}^{n} \xi_{k} x \mathbf{1}_{\mathbb{S}_{t_{k}}^{3}}(\tau, w)\right)\right) \mu_{\varepsilon}(\mathrm{d} x, \mathrm{~d} x, \mathrm{~d} \tau, \mathrm{d} w)\right) .
\end{aligned}
$$

Using $|\exp (i x)-1-i x| \leq C x^{2}$, we have

$$
\int_{E} \frac{1}{\varepsilon^{2}}\left(\exp \left(i \varepsilon \sum_{k=1}^{n} \xi_{k} x \mathbf{1}_{\mathbb{S}_{t_{k}}^{3}}(\tau, w)\right)-1-i \varepsilon \sum_{k=1}^{n} \xi_{k} x \mathbf{1}_{\mathbb{S}_{t_{k}}^{3}}(\tau, w)\right) \leq C x^{2} \mathbf{1}_{\mathbb{S}_{t_{k}}^{3}}(\tau, w) .
$$

As $C x^{2} \mathbf{1}_{\mathbb{S}_{t}^{3}}(\tau, w)$ is integrable with respect to $\prod_{i=1}^{d} w_{i}^{-1-\theta} F\left(x, x^{\prime}\right) \mathrm{d} w \mathrm{~d} \tau \mathrm{d} x \mathrm{~d} x^{\prime}$, we can apply the Lebesgue dominated convergence theorem to obtain

$$
\lim _{\varepsilon \rightarrow 0} \psi_{\varepsilon}\left(\xi_{1}, \ldots, \xi_{n}\right)=\exp \left(-\frac{\mathrm{E}\left[X^{2}\right]}{2} \int_{\mathbb{R}^{d} \times \mathbb{R}_{+}^{d}}\left(\sum_{k=1}^{n} \xi_{k} \mathbf{1}_{\mathbb{S}_{t_{k}}^{3}}(\tau, w)\right)^{2} \frac{1}{2} p_{0}^{\prime}(\mathrm{d} \tau, \mathrm{d} w)\right) .
$$

Recall that $p_{0}^{\prime}=\bigotimes_{i=1}^{d} p_{0}$. Expanding the product, we obtain

$$
\begin{aligned}
& \lim _{\varepsilon \rightarrow 0} \psi_{\varepsilon}\left(\xi_{1}, \ldots, \xi_{n}\right) \\
& \quad=\exp \left(-\frac{\mathrm{E}\left[X^{2}\right]}{4} \sum_{k=1}^{n} \sum_{l=1}^{n} \xi_{k} \xi_{l} \int_{\mathbb{R} \times \mathbb{R}_{+}} \mathbf{1}_{\mathbb{S}_{t_{k}}^{3}}(\tau, w) \mathbf{1}_{\mathbb{S}_{t_{l}}^{3}}(\tau, w) p_{0}^{\prime}(\mathrm{d} \tau, \mathrm{d} w)\right) .
\end{aligned}
$$

As we have

$$
\mathbb{S}_{t_{k}}^{3} \cap \mathbb{S}_{t_{l}}^{3}=\left\{(\tau, w): \text { for all } i,-\min \left(t_{k}^{i}, t_{l}^{i}\right) \leq \tau<\min \left(t_{k}^{i}, t_{l}^{i}\right), \max \left(t_{k}^{i}, t_{l}^{i}\right) \leq \tau+w\right\},
$$


using Lemma A.2, we obtain

$$
p_{0}^{\prime}\left(\mathbb{S}_{t_{k}}^{3} \cap \mathbb{S}_{t_{l}}^{3}\right)=\frac{1}{\theta^{d}(1-\theta)^{d}} \prod_{i=1}^{d}\left(\left|t_{k}^{i}+t_{l}^{i}\right|^{1-\theta}-\left|t_{k}^{i}-t_{l}^{i}\right|^{1-\theta}\right) .
$$

Using this in (3.1) yields

$$
\lim _{\varepsilon \rightarrow 0} \psi_{\varepsilon}\left(\xi_{1}, \ldots, \xi_{n}\right)=\exp \left(-\frac{\mathrm{E}\left[X^{2}\right]}{4 \theta^{d}(1-\theta)^{d}} \sum_{k=1}^{n} \sum_{l=1}^{n} \xi_{k} \xi_{l} \prod_{i=1}^{d}\left(\left|t_{k}^{i}+t_{l}^{i}\right|^{1-\theta}-\left|t_{k}^{i}-t_{l}^{i}\right|^{1-\theta}\right)\right) .
$$

We recognize the covariance function of a bifractional Brownian sheet, which means that the finite-dimensional laws of $\breve{Z}_{\varepsilon}^{3}$ converge to those of a BBM with parameters $\left(\frac{1}{2}, 1-\theta\right)$ times $\sqrt{\left(2 \theta^{d}(1-\theta)^{d}-1\right) / \mathrm{E}\left[X^{2}\right]}$.

Since the difference between two independent symmetric Gaussian processes with the same distribution is up to a multiplicative constant of $\sqrt{2}$ a process with the same distribution, and since $\breve{Z}_{\varepsilon}^{3}$ and $\breve{Z}_{\varepsilon}^{1}$ converge to independent processes with the same distribution, the finitedimensional laws of $\breve{Z}_{\varepsilon}^{3}-\breve{Z}_{\varepsilon}^{1}$ converge to those of a BBM with parameters $\left(\frac{1}{2}, 1-\theta\right)$ times $\sqrt{2 \theta^{d}(1-\theta)^{d} / \mathrm{E}\left[X^{2}\right]}$.

(b) We will compute the characteristic function of the process $\varepsilon^{d \theta / 2}\left(\breve{Y}_{\varepsilon}(t)-\mathrm{E}\left[\breve{Y}_{\varepsilon}\right]\right)$. Take $\xi_{1}, \ldots, \xi_{n} \in \mathbb{R}$ and $t_{1}, \ldots, t_{n} \in \mathbb{R}^{d}$ with $t_{i}=\left(t_{i}^{1}, \ldots, t_{i}^{d}\right)$. We have

$$
\begin{aligned}
& \psi_{\varepsilon}\left(\xi_{1}, \ldots, \xi_{n}\right) \\
&= \mathrm{E}\left[\exp \left(\left(i \sum_{k=1}^{n} \xi_{k} \varepsilon^{d \theta / 2} \breve{Y}_{\varepsilon}\left(t_{k}\right)\right)-\mathrm{E}\left[i \sum_{k=1}^{n} \xi_{k} \varepsilon^{d \theta / 2} \breve{Y}_{\varepsilon}\left(t_{k}\right)\right]\right)\right] \\
&= \mathrm{E}\left[\operatorname { e x p } i \varepsilon ^ { d \theta / 2 } \int _ { E } \varepsilon \sum _ { k = 1 } ^ { n } \xi _ { k } \left(x\left(\mathbf{1}_{\mathbb{S}_{t_{k}}^{2}}(\tau, w)+\mathbf{1}_{\mathbb{S}_{t_{k}}^{3}}(\tau, w)\right)\right.\right. \\
&\left.\left.-x^{\prime}\left(\mathbf{1}_{\mathbb{S}_{t_{k}}^{2}}(\tau, w)+\mathbf{1}_{\mathbb{S}_{t_{k}}^{1}}(\tau, w)\right)\right) N_{\varepsilon}\left(\mathrm{d} x, \mathrm{~d} x^{\prime}, \mathrm{d} \tau, \mathrm{d} w\right)\right] \\
& \times \exp \mathrm{E}\left[-i \sum_{k=1}^{n} \xi_{k} \varepsilon^{d \theta / 2} \breve{Y}_{\varepsilon}\left(t_{k}\right)\right] .
\end{aligned}
$$

Following the same steps as in the proof of Theorem 2.1, we obtain

$$
\begin{gathered}
\psi_{\varepsilon}\left(\xi_{1}, \ldots, \xi_{n}\right)=\exp \left[\int _ { E } \left(\operatorname { e x p } \left(i \varepsilon ^ { ( d \theta + 2 ) / 2 } \sum _ { k = 1 } ^ { n } \xi _ { k } \left(x\left(\mathbf{1}_{\mathbb{S}_{t_{k}}^{2}}(\tau, w)+\mathbf{1}_{\mathbb{S}_{t_{k}}^{3}}(\tau, w)\right)\right.\right.\right.\right. \\
\left.\left.-x^{\prime}\left(\mathbf{1}_{\mathbb{S}_{t_{k}}^{2}}(\tau, w)+\mathbf{1}_{\mathbb{S}_{t_{k}}^{1}}(\tau, w)\right)\right)\right) \\
-1-i \varepsilon^{(d \theta+2) / 2} \sum_{k=1}^{n} \xi_{k}\left(x\left(\mathbf{1}_{\mathbb{S}_{t_{k}}^{2}}(\tau, w)+\mathbf{1}_{\mathbb{S}_{t_{k}}^{3}}(\tau, w)\right)\right. \\
\left.\left.\quad \times \mu_{\varepsilon}^{\prime}\left(\mathbf{1}_{\mathbb{S}_{t_{k}}^{2}}(\tau, w)+\mathbf{1}_{\mathbb{S}_{t_{k}}^{1}}(\tau, w)\right)\right)\right) \\
\end{gathered}
$$


Again, the Lebesgue dominated convergence theorem cannot be used here because the integral is not convergent when $w_{j} \rightarrow 0$. Define

$$
f\left(x, x^{\prime}, \tau, w\right)=\sum_{k=1}^{n} \xi_{k}\left(x\left(\mathbf{1}_{\mathbb{S}_{t_{k}}^{2}}(\tau, w)+\mathbf{1}_{\mathbb{S}_{t_{k}}^{3}}(\tau, w)\right)-x^{\prime}\left(\mathbf{1}_{\mathbb{S}_{t_{k}}^{2}}(\tau, w)+\mathbf{1}_{\mathbb{S}_{t_{k}}^{1}}(\tau, w)\right)\right)
$$

and

$$
g_{\varepsilon}\left(x, x^{\prime}, \tau, w\right)=\frac{1}{\varepsilon^{2+d \theta}}\left(\mathrm{e}^{i \varepsilon^{(d \theta+2) / 2} f}-1-i \varepsilon^{(d \theta+2) / 2} f\right)
$$

so that

$$
\psi_{\varepsilon}\left(\xi_{1}, \ldots, \xi_{n}\right)=\int_{E} \varepsilon^{d \theta} g_{\varepsilon} \prod_{i=1}^{d} w_{i}^{-1-\theta} \mathrm{d} w_{i} \mathrm{~d} x \mathrm{~d} x^{\prime} \mathrm{d} \tau
$$

Furthermore, define

$$
h\left(x, x^{\prime}, \tau, w\right)=\sum_{k=1}^{n} \sum_{l=1}^{n} \xi_{k} \xi_{l}\left(x-x^{\prime}\right)^{2} \mathbf{1}_{\mathbb{S}_{t_{l}}^{2} \cap \mathbb{S}_{t_{k}}^{2}}(\tau, w)
$$

and consider

$$
\int_{E}\left(\varepsilon^{\theta} g_{\varepsilon}\left(x, x^{\prime}, \tau, w\right)+\frac{1}{2} \varepsilon^{\theta} h\left(x, x^{\prime}, \tau, w\right)\right) \mathbf{1}_{\{w>\varepsilon\}} w^{-1-\theta} F\left(\mathrm{~d} x, \mathrm{~d} x^{\prime}\right) \mathrm{d} \tau \mathrm{d} w .
$$

Note that

$$
\left|\varepsilon^{d \theta} g_{\varepsilon}\left(x, x^{\prime}, \tau, w\right)+\frac{1}{2} \varepsilon^{d \theta} h\left(x, x^{\prime}, \tau, w\right)\right| \leq \varepsilon^{d \theta}\left|g_{\varepsilon}+\frac{1}{2} f^{2}\right|+\frac{\varepsilon^{d \theta}}{2}\left|f^{2}-h\right| .
$$

On the one hand, we have

$$
g_{\varepsilon}+\frac{1}{2} f^{2}=\frac{1}{\varepsilon^{d \theta+2}}\left(\mathrm{e}^{i \varepsilon^{(d \theta+2) / 2} f}-1-i \varepsilon^{(d \theta+2) / 2} f+\frac{\varepsilon^{d \theta+2}}{2} f^{2}\right),
$$

which implies that

$$
\varepsilon^{d \theta}\left|g_{\varepsilon}+\frac{1}{2} f^{2}\right| \leq C \varepsilon^{3 d \theta / 2+1}|f|^{3} .
$$

According to Lemma A.3 in Appendix A,

$$
\int_{E}\left|f^{3}\right| \prod_{i=1}^{d} w_{i}^{-1-\theta} \mathbf{1}_{\left\{w_{i}>\varepsilon\right\}} \mathrm{d} w_{i} \mathrm{~d} x \mathrm{~d} x^{\prime} \mathrm{d} \tau \leq \varepsilon^{d \theta} C_{t_{1}, \ldots, t_{n}}
$$

where $C_{t_{1}, \ldots, t_{n}}$ is a constant depending on $t_{1}, \ldots, t_{n}$ and not on $\varepsilon$. On the other hand, expanding $f^{2}-h$, the terms giving the $\varepsilon^{d \theta}$ vanish, which implies that

$$
\int_{E}\left|f^{2}-h\right| \prod_{i=1}^{d} w_{i}^{-1-\theta} \mathbf{1}_{\left\{w_{i}>\varepsilon\right\}} \mathrm{d} w_{i} \mathrm{~d} x \mathrm{~d} x^{\prime} \mathrm{d} \tau \leq \varepsilon^{(d-1) \theta} C_{t_{1}, \ldots, t_{n}}^{\prime},
$$

where $C_{t_{1}, \ldots, t_{n}}$ is another constant depending on $t_{1}, \ldots, t_{n}$ and not on $\varepsilon$. 
Using the two upper bounds in (3.3) and integrating, we obtain

$$
\lim _{\varepsilon \rightarrow 0} \int_{E}\left(\varepsilon^{d \theta} g_{\varepsilon}\left(x, x^{\prime}, \tau, w\right)+\frac{1}{2} \varepsilon^{d \theta} h\left(x, x^{\prime}, \tau, w\right)\right) \prod_{i=1}^{d} w_{i}^{-1-\theta} \mathbf{1}_{\left\{w_{i}>\varepsilon\right\}} \mathrm{d} w_{i} \mathrm{~d} x \mathrm{~d} x^{\prime} \mathrm{d} \tau=0 .
$$

In other words,

$$
\begin{aligned}
& \lim _{\varepsilon \rightarrow 0} \int_{E} \varepsilon^{d \theta} g_{\varepsilon}\left(x, x^{\prime}, \tau, w\right) \prod_{i=1}^{d} w_{i}^{-1-\theta} \mathbf{1}_{\left\{w_{i}>\varepsilon\right\}} \mathrm{d} w_{i} \mathrm{~d} x \mathrm{~d} x^{\prime} \mathrm{d} \tau \\
& \quad=\lim _{\varepsilon \rightarrow 0}-\frac{\varepsilon^{d \theta}}{2} \int_{E} h\left(x, x^{\prime}, \tau, w\right) \prod_{i=1}^{d} w_{i}^{-1-\theta} \mathbf{1}_{\left\{w_{i}>\varepsilon\right\}} \mathrm{d} w_{i} \mathrm{~d} x \mathrm{~d} x^{\prime} \mathrm{d} \tau
\end{aligned}
$$

Using Lemma A.2 in Appendix A, we obtain

$$
\begin{aligned}
& \lim _{\varepsilon \rightarrow 0} \int_{E} \varepsilon^{d \theta} g_{\varepsilon} \prod_{i=1}^{d} w_{i}^{-1-\theta} \mathbf{1}_{\left\{w_{i}>\varepsilon\right\}} \mathrm{d} w_{i} \mathrm{~d} x \mathrm{~d} x^{\prime} \mathrm{d} \tau \\
& \quad=-\frac{2^{d} \mathrm{E}\left[\left(X-X^{\prime}\right)^{2}\right]}{4 \theta^{d}} \sum_{k=1}^{n} \sum_{l=1}^{n} \xi_{k} \xi_{l} \prod_{i=1}^{d} \min \left(t_{k}^{i}, t_{l}^{i}\right) .
\end{aligned}
$$

Using (3.2) yields the conclusion.

Note that Theorem 3.1 is not a generalization of Theorem 2.1, but a modification which ensures a better-known limit process. To establish a theorem from which Theorem 2.1 is a special case for $d=1$, we would have to

- use the same Poisson process $\Pi_{\varepsilon}^{\prime}$ defined in this section,

- consider the sets $\bar{S}_{t}^{j}=\prod_{i=1}^{d} S_{t_{i}}^{j}$ defined for $t=\left(t_{1}, \ldots, t_{d}\right) \in \mathbb{R}_{+}^{d}$ and $j=1,2,3$, where the sets $S_{t_{i}}^{j}$ are given in (2.1a)-(2.1e).

Denote by $\bar{Y}_{\varepsilon}$ the process defined by

$$
\bar{Y}_{\varepsilon}(t)=\sum_{j \in \mathbb{N}} \varepsilon\left(X_{j} \mathbf{1}_{\left(\tau_{j}, w_{j}\right) \in \bar{S}_{t}^{3}}-X_{j}^{\prime} \mathbf{1}_{\left(\tau_{j}, w_{j}\right) \in \bar{S}_{t}^{1}}+\left(X_{j}-X_{j}^{\prime}\right) \mathbf{1}_{\left(\tau_{j}, w_{j}\right) \in \bar{S}_{t}^{2}}\right),
$$

where $\left(X_{j}, X_{j}^{\prime}, \tau_{j}, w_{j}\right)_{j \in \mathbb{N}}$ is an enumeration of the Poisson point process $\Pi_{\varepsilon}^{\prime}$.

Then we can obtain the following result using the same methods.

Theorem 3.2. (a) If $X=X^{\prime}$ almost surely then $\varepsilon^{(d-1) \theta / 2} \bar{Y}_{\varepsilon}$ converges in distribution to a centered Gaussian process whose covariance function for $t=\left(t^{1}, \ldots, t^{d}\right) \in \mathbb{R}_{+}^{d}$ and $s=$ $\left(s^{1}, \ldots, s^{d}\right) \in \mathbb{R}_{+}^{d}$ is given by

$$
r(s, t)=\frac{\mathrm{E}\left[X^{2}\right]}{4 \theta^{d}(1-\theta)^{d}}\left(\prod_{i=1}^{d}\left(\max \left(t^{i}, s^{i}\right)^{1-\theta}-\left|t^{i}-s^{i}\right|^{1-\theta}\right)+\prod_{i=1}^{d} \min \left(t^{i}, s^{i}\right)^{1-\theta}\right),
$$

which is an extension of FBM in higher dimensions. 
(b) If $X \neq X^{\prime}$ on a nonnegligible set, the process $\varepsilon^{d \theta / 2}\left(\bar{Y}_{\varepsilon}(t)-\mathrm{E}\left[\bar{Y}_{\varepsilon}\right]\right)$ converges in distribution to

$$
\sqrt{\frac{2^{d-1} \mathrm{E}\left[\left(X-X^{\prime}\right)^{2}\right]}{\theta^{d}}} B
$$

where $B$ is a Brownian sheet.

Unfortunately, the limit process obtained is not as interesting as the bifractional Brownian sheet and the proof is similar, so we omit the details.

Remark 3.1. This model and others with similar behaviors are studied in detail in the author's $\mathrm{PhD}$ thesis [13].

\section{Appendix A. Lemmas}

This appendix contains a lemma used to prove tightness in the proof of Theorem 2.1. To this end, we will compute moments of a process which is the sum of a function over a Poisson process. Denote by $\Pi$ a Poisson process on a space $E$. If $f$ is a real-valued function defined on $E$, the sum of $f$ over $\Pi$ is

$$
\Sigma=\sum_{x \in \Pi} f(x)
$$

If we denote by $N$ the random measure associated with $\Pi$, i.e. $N=\sum_{x \in \Pi} \delta_{x}$, we can also write

$$
\Sigma=\int_{E} f(x) N(\mathrm{~d} x) .
$$

Denote by $\mu$ the intensity measure of $\Pi$, i.e. the measure on $E$ such that $\mu(A)=\mathrm{E}[N(A)]$ for all $A$ measurable sets in $E$. Lemma A.1 below was not found in the literature, but it can be proven following the leads found in Chapter 3 of [10].

Lemma A.1. Let $\Pi$ be a Poisson process of intensity measure $\mu$ on a space E. Take $n \in \mathbb{N}^{*}$. Let $f_{1}$ and $f_{2}$ be two real-valued functions over $E$ such that, for all $k \in \mathbb{N}$,

$$
\int_{E} \max \left(\left|f_{1}\right|,\left|f_{2}\right|\right)^{k} \mathrm{~d} \mu<\infty .
$$

Define $\Sigma_{1}=\sum_{X \in \Pi} f_{1}(X)$ and $\Sigma_{2}=\sum_{X \in \Pi} f_{2}(X)$. Then,

$$
\mathrm{E}\left[\left(\Sigma_{1}-\mathrm{E} \Sigma_{1}\right)^{n}\left(\Sigma_{2}-\mathrm{E} \Sigma_{2}\right)^{n}\right]=\frac{(2 n) !}{\left(\begin{array}{c}
2 n \\
n
\end{array}\right)} \sum_{k, l \in \delta_{n}} \prod_{i=1}^{d} \frac{\left(\begin{array}{c}
k_{i}+l_{i} \\
l_{i}
\end{array}\right)}{d !\left(k_{i}+l_{i}\right) !} I_{l_{i}, k_{i}},
$$

where

$$
\S_{n}=\bigcup_{d=1}^{n}\left\{k, l \in \mathbb{N}^{d}, \sum_{i=1}^{d} k_{i}=\sum_{i=1}^{d} l_{i}=n \text { and } k_{i}+l_{i} \geq 2\right\}
$$

and $I_{l_{i}, k_{i}}=\int_{E} f_{1}^{l_{i}} f_{2}^{k_{i}} \mathrm{~d} \mu$.

The following two technical lemmas are often used in the paper to compute integrals. Recall the measure $p_{\varepsilon}=w^{-1-\theta} \mathbf{1}_{\{w>\varepsilon\}} \mathrm{d} \tau \mathrm{d} w$ defined on $\mathbb{R} \times \mathbb{R}_{+}$. Lemma A.2 below is often used to prove the convergence of finite-dimensional laws, whereas Lemma A.3 below is helpful to prove tightness.

In the following, we will define $a \vee b=\max (a, b)$ and $a \wedge b=\min (a, b)$. Both the following lemmas are proved with straightforward computations, so we will omit their proofs. 
Lemma A.2. (a) Let $a, b, c, d \in \mathbb{R}$ be such that $a<b, c<d$, $a \leq c$, and $b \leq d$. We have

$$
\begin{aligned}
p_{\varepsilon}(a \leq & \tau<b, c \leq \tau+w<d) \\
= & \frac{\varepsilon^{-\theta}}{\theta}((c-a) \wedge \varepsilon-(c-b) \wedge \varepsilon-(d-a) \wedge \varepsilon+(d-b) \wedge \varepsilon) \\
& +\frac{1}{\theta(1-\theta)}\left(((c-a) \vee \varepsilon)^{1-\theta}-((c-b) \vee \varepsilon)^{1-\theta}\right. \\
& \left.-((d-a) \vee \varepsilon)^{1-\theta}+((d-b) \vee \varepsilon)^{1-\theta}\right) .
\end{aligned}
$$

(b) If $d=\infty$,

$$
\begin{aligned}
p_{\varepsilon}(a \leq \tau<b, c \leq \tau+w)= & \frac{\varepsilon^{-\theta}}{\theta}((c-a) \wedge \varepsilon-(c-b) \wedge \varepsilon) \\
& +\frac{1}{\theta(1-\theta)}\left(((c-a) \vee \varepsilon)^{1-\theta}-((c-b) \vee \varepsilon)^{1-\theta}\right)
\end{aligned}
$$

(c) If $a=-\infty$,

$$
\begin{aligned}
p_{\varepsilon}(\tau<b, c \leq \tau+w<d)= & \frac{\varepsilon^{-\theta}}{\theta}((d-b) \wedge \varepsilon-(c-b) \wedge \varepsilon) \\
& +\frac{1}{\theta(1-\theta)}\left(((d-b) \vee \varepsilon)^{1-\theta}-((c-b) \vee \varepsilon)^{1-\theta}\right)
\end{aligned}
$$

(d) If two numbers among $a, b, c$, and d are infinite, $p_{\varepsilon}(a \leq \tau<b, c \leq \tau+w<d)=\infty$.

Remark A.1. Note that $p_{\varepsilon}$ does not charge $\{a \leq \tau<b, c \leq \tau+w<d\}$ if $a \geq d$ because $p_{\varepsilon}$ only charges sets where $w>0$. This is why we assumed that $b \leq d$ and $a \leq c$ in Lemma A.2.

Lemma A.3. (a) Let $a, b, c, d \in \mathbb{R}$ be such that $a<b, c<d$, $a \leq c$, and $b \leq d$. We have

$$
p_{\varepsilon}(a \leq \tau<b, c \leq \tau+w<d) \leq \frac{2}{\theta} \min (b-a, d-c)((c-b) \vee \varepsilon)^{-\theta} .
$$

In some cases, we can even have a bound depending on $d-a$ : if $d-a \leq \varepsilon$ then

$$
p_{\varepsilon}(a \leq \tau<b, c \leq \tau+w<d)=0 .
$$

Furthermore, if $b=c$, we have

$$
p_{\varepsilon}(a \leq \tau<b, b \leq \tau+w<d) \leq \frac{(d-a)^{1-\theta}}{\theta(1-\theta)} .
$$

(b) If $d=\infty$,

$$
p_{\varepsilon}(a \leq \tau<b, c \leq \tau+w) \leq \frac{b-a}{\theta}((c-b) \vee \varepsilon)^{-\theta}
$$

(c) If $a=-\infty$,

$$
p_{\varepsilon}(\tau<b, c \leq \tau+w<d)=\frac{d-c}{\theta}((c-b) \vee \varepsilon)^{-\theta} .
$$




\section{Acknowledgement}

The author would like to thank Clément Dombry for the idea that initiated this paper.

\section{References}

[1] Biermé, H. and Estrade, A. (2006). Poisson random balls: self-similarity and x-ray images. Adv. Appl. Prob. 38, 853-872.

[2] Biermé, H., Estrade, A. AND KaJ, I. (2010). Self-similar random fields and rescaled random balls models. J. Theoret. Prob. 23, 1110-1141.

[3] Billingsley, P. (1999). Convergence of Probability Measures, 2nd edn. John Wiley, New York.

[4] Breton, J.-C. AND Dombry, C. (2009). Rescaled weighted random ball models and stable self-similar random fields. Stoch. Process. Appl. 119, 3633-3652.

[5] Cioczek-Georges, R. and Mandelbrot, B. B. (1995). A class of micropulses and antipersistent fractional Brownian motion. Stoch. Process. Appl. 60, 1-18.

[6] Cioczek-Georges, R. and Mandelbrot, B. B. (1996). Alternative micropulses and fractional Brownian motion. Stoch. Process. Appl. 64, 143-152.

[7] Houdré, C. And Villa, J. (2003). An example of infinite dimensional quasi-helix. In Stochastic Models (Mexico City, 2002; Contemp. Math. 336), American Mathematical Society, Providence, RI, pp. 195-201.

[8] KaJ, I., Leskelä, L., Norros, I. And Schmidt, V. (2007). Scaling limits for random fields with long-range dependence. Ann. Prob. 35, 528-550.

[9] Kallenberg, O. (2002). Foundations of Modern Probability, 2nd edn. Springer, New York.

[10] Kingman, J. F. C. (1993). Poisson Processes (Oxford Stud. Prob. 3). Oxford University Press, New York.

[11] Kolmogorov, A. (1940). Wienersche Spiralen und einige andere interessante Kurven im Hilbertschen Raum. C. R. (Doklady) Acad. URSS (NS) 26, 115-118.

[12] Mandelbrot, B. B. And Van Ness, J. W. (1968). Fractional Brownian motions, fractional noises and applications. SIAM Rev. 10, 422-437.

[13] Marouby, M. (2010). Trois études de processus fractionnaires. Doctoral Thesis, Université Paul Sabatier. Available at http://thesesups.ups-tlse.fr/946/1/Marouby_Matthieu.pdf.

[14] Tudor, C. A. And XiaO, Y. (2007). Sample path properties of bifractional Brownian motion. Bernoulli 13, 1023-1052. 